\title{
THE SUBSTITUTION OF A TETRAHEDRON FOR THE EINTHOVEN TRIANGLE
}

Frank N. Wilson and Franklin D. Johnston, Ann Arbor, Mich., and Charles E. Kossmann, New York, N. Y.

$\mathrm{T}$ HE early studies of the electrical phenomena associated with the human heartbeat carried out by Waller and by Einthoven and his associates led to the adoption of the leads from the two arms and the left leg which are still in universal use. For a great many years little thought was given to the possibility that these leads might be entirely inadequate in certain respects and that great progress in electrocardiographic diagnosis might be achieved by supple. menting them with leads of other kinds. Some fifteen years ago, however, it became evident that leads in which one electrode is placed on the precordium and the other on some part of the body much farther from the heart are, to some extent, similar to unipolar leads from the ventricular surface, such as were used by Lewis and Rothschild, ${ }^{1}$ and are capable of yielding information which limb leads cannot give. In the last decade, precordial leads have become indispensable.

This sequence of events raises the question as to whether further important advances in elcctrocardiography are likely to result from the development of still other new leads. If, like our predecessors, we may be overlooking opportunities in this direction, it is worth while to examine our present situation in the hope of ascertaining how we should proceed in order to take advantage of any such that may exist.

The general character of the heart's electrical field and the main features of the relations between it and the rise and decline of the excitatory process were clearly understood by Waller, by Einthoven, and by some of their contemporaries. These pioneers realized that, for certain purposes, the electromotive force of the heart may be regarded as a vector and that the limb leads are poorly suited to the study of the cardioelectric forces which are perpendicular to the plane defined by them. They knew also that these leads, unlike those from electrodes in contact with the heart's surface, yield a kind of average electrocardiogram which cannot be expected to depict variations in the excitatory process that involve only a small region of cardiac muscle and leave the general course of myocardial activation and recovery unchanged. At the start, it was, however, naturally difficult to interpret experiments in which direct leads from the ventricular surface were employed; the more so because of the inconstancy and variability of the form of the ventricular complex encountered in leads of this kind. These

From the Department of Internal Medicine, University of Michigan Medical School. Read at the Inter-American Congress of Cardiology, Mexico, D. F., Oct. 5-12, 1946. 
variations were troublesome even though the factors responsible for them were understood. This probably explains why indirect leads came to be preferred to direct leads in experiments on animals in which the latter would have been much more useful.

The history of the development of our knowledge of electrocardiographic leads indicates that two courses are open to us in our attempts to find better methods of leading than we now possess. The first is to search for additional leads akin to unipolar precordial leads. These may be regarded as semidirect leads from the anterior ventricular surface. Such leads have a particularly favorable spatial relation to the cardiac muscle nearest the exploring electrode and they are capable of detecting lesions affecting it which are far outside the reach of any lead or combination of leads from electrodes that are distant from the heart. On the other hand, they are clearly unsuitable for discovering lesions so located that the exploring electrode cannot be placed much closer to them than to more normal parts of the myocardium. Since a semidirect lead from one cardiac region is necessarily an indirect lead from others, it is obvious that an adequate study of the whole heart by this method must involve the use of many leads. A relatively small exploring electrode must also be employed, for if a large one is used, the advantages gained by putting it close to the heart are lost through its short-circuiting effect.

Unfortunately, most of the epicardial surface is not accessible to exploration by semidirect leads. As far as the anterior ventricular surface is concerned, we can do fairly well by moving the exploring electrode step by step across the precordium, but the posterior ventricular surface is much farther from the skin so that unipolar leads from the back are far less effective than unipolar leads from the front of the chest. Unipolar esophageal leads are very useful for the purpose of studying certain auricular disturbances and often furnish important information in cases of posterior infarction. For the purpose of exploring all of the posterior ventricular wall they leave much to be desired. Worth-while information will certainly be gained by taking unipolar leads from the cavities of the right auricle and right ventricle which are accessible by catheterization. This procedure will surely prove a valuable one for research and may occasionally yield data of clinical value. On the whole, however, undeveloped possibilities of improving cardiac diagnosis through the exploration of additional parts of the epicardial surface by means of semidirect unipolar leads do not seem particularly promising.

The second course is to try to find the indirect leads that will give us the most useful over-all or average electrocardiograms with the full realization that such leads cannot be expected to supply information regarding abnormalities of the excitatory process that do not involve comparatively large regions of muscle. It is clear that the leads needed for this purpose will not be numerous so that the time required to take them will be small. The electrodes employed may be relatively large, and the use of such electrodes reduces to a minimum the difficulties due to the high resistance of the skin and to polarization. Neither the standard limb leads nor unipolar limb leads adequately meet our needs because they do not furnish sufficient information concerning variations in the excitatory 
process affecting chiefly the sagittal components of the cardiac electromotive forces. By means of limb leads alone it is impossible to detect lesions confined to certain parts of the heart muscle and very difficult to differentiate peculiarities of the form of the electrocardiogram due to rotation of the heart about an axis that is nearly parallel to the frontal plane from those due to intrinsic cardiac disturbances.

Einthoven's early papers display his deep interest in the effects of rotation of the heart upon the electrocardiographic deflections and his method of finding the projection of the electrical axis of the heart upon the frontal plane was evidently an outgrowth of ideas that he had begun to develop years earlier. Many since his day have been equally conscious of this problem and have made important contributions bearing upon it. Nevertheless, much remains to be done. We have reached the stage where we know that rotation of the heart often has profound effects upon the form of the electrocardiogram which we have in the past attributed to other causes, but our ability to recognize these effects with certainty is still small.

The possibility of improving this situation by employing sagittal leads of one kind or another has occurred to many investigators. We regret that it is not possible to review the extensive literature on this subject. We shall refer only to the work of Arrighi, ${ }^{2}$ of Buenos Aires, In his doctoral thesis, Arrighi analyzed a large series of trarings obtained by means of three leads which define a sagittal triangle. The apices of this triangle are a point on the left submaxillary region close to the chin, a point 3 or $4 \mathrm{~cm}$. to the left of the midpoint of a line joining the umbilicus with the center of the pubis, and a point in the left interscapular region and approximately at the level of the spinous process of the seventh thoracic vertebra. Arrighi showed that his leads and the standard limb leads give the same value for the vertical component of the cardiac vector. He advanced cogent arguments in support of his contention that his leads permit an accurate estimate of the sagittal component of this vector. Our only criticism of his approach to the problem is that it involves the taking of three extra leads when only one should be required.

The fact remains that no method of determining the spatial electrical axis of the heart has been very extensively used either by him who originated it or by anyone else. One rcason lies in the difficulty of measuring the error involved in any such method that is proposed and of demonstrating that by such means effects produced by rotation of the heart can be recognized with certainty. It seems probable that these difficulties will eventually be overcome.

\section{DFSCRIPTION OF AN EXPERIMENT ON A CADAVER}

On March 1, 1934, we carried out an experiment on a cadavar for the purpose of ascertaining whether, by converting Einthoven's triangle into a tetrahedron, we could devise a reliable method of computing the position of the spatial electrical axis of the heart. The results of this single experiment, performed so long ago, are reported now chicfly for the purpose of laying the groundwork for future papers dealing with more recent work along the same line, and also because 
they have a bearing upon the validity of Einthoven's triangle which has been the subject of a good deal of discussion.

The cadaver used was that of a man who had died more than a week before of carcinoma of the face complicated by pneumonia. During the interim the body had lain in the morgue in the supine posture, and it was suspected that, in addition to pronounced post-mortem changes, there had been considerable gravitation of fluid into the more dorsal tissues. The electrodes used to generate an electric field in the trunk consisted of two brass rods $(25 \mathrm{~cm}$. long) covered with rubber except at the sharpened tips. They were fixed in a wooden frame which kept them parallel and $5 \mathrm{~cm}$. apart. This frame permitted the rods to be moved endwise, so that when they were thrust through the precordium, the depth of the tip of each was independently adjustable. After they were in place a potential difference of approximately 18 volts was rhythmically impressed upon these electrodes by means of a rotating contact breaker. Small copper disks with central binding posts sewn beneath the skin with the binding post projecting through it served as electrodes for the leads employed. One was placed on the lateral surface of each arm at the level of the insertion of the deltoid muscle and another on the inner aspect of the left knee joint. The fourth was placed on the back just to the left of the midline and at the level of the spinous process of the seventh thoracic vertebra.

The leads taken were standard Leads I, II, and III and leads from a central terminal to each of the four electrodes. These last leads will be referred to as Leads $V_{R}, V_{L}, V_{F}$, and $V_{B}$, respectively. On most occasions when these unipolar leads were taken, resistances of $10,000 \mathrm{ohms}$ were used to connect the central terminal to the limb and back electrodes. Once 5,000 ohm resistances were employed. In a few instances the resistance between the central terminal and the back electrode was increased to 15,000 or $20,000 \mathrm{ohms}$ or was removed. The effects of shifting the arm electrodes to the anteromedial margin of the shoulder joints and the leg electrode to a point just above the pubis were also investigated.

During the course of the experiment the electrodes used to generate the field occupied four different positions as follows:

Position $A$. Both electrodes in the third intercostal space. The minus electrode was close to the right and the plus electrode close to the left border of the sternum. The tip of the former was $5.7 \mathrm{~cm}$. and that of the latter $8.8 \mathrm{~cm}$. beneath the skin. The line joining these made, therefore, an angle of 32 degrees with the frontal plane. Its projection on this plane was parallel to the line of Lead I. The tips of the electrodes were $5.9 \mathrm{~cm}$. apart, and their mean distance from the front of the chest was 7.25 centimeters.

Position B. Same as Position A except that the depth of the minus electrode was $5 \mathrm{~cm}$. and that of the plus electrode 10 centimeters. The line joining their tips made an angle of 45 degrees with the frontal plane. This line was $7.1 \mathrm{~cm}$. long and the depth of its midpoint was 7.5 centimeters.

Position C. The minus electrode was in the fourth intercostal space and the plus electrode in the third. Both were close to the left edge of the sternum. The tip of the first was $5.5 \mathrm{~cm}$. and that of the second $10.7 \mathrm{~cm}$. beneath the skin. 
The line joining these tips was $7.2 \mathrm{~cm}$. long and the depth of its midpoint was 8.1 centimeters. The angle made by it with the frontal plane was 46 degrees. Its projection on this plane was not quite perpendicular to the line of Lead I.

Position D. Same as Position C except that the tip of the minus electrode was $5.5 \mathrm{~cm}$. and that of the plus electrode $15 \mathrm{~cm}$. beneath the skin. The line joining the uninsulated tips made an angle of 62 degrees with the frontal plane. It was $10.7 \mathrm{~cm}$. long and its midpoint was at a depth of 10.25 centimeters. The diameter of the chest from front to back was about $21 \mathrm{~cm}$. so that the plus electrode was not more than $6 \mathrm{~cm}$. from its posterior surface.

The measurements of the deflections in the various leads are given in Table $I$. Figures enclosed in parentheses were computed from those given in the other columns. Einthoven's $E$ and his angle $\alpha$ were derived from the deflections in the standard limb leads in the usual way. At the time when the experiment was performed and before the results were known, the true angle $\alpha$ defined by the line joining the points where the input electrodes penetrated the anterior wall of the chest and the direction of Lead I was estimated at 0 degree for Positions A and $\mathrm{B}$ at -100 degrees for Positions $\mathrm{C}$ and $\mathrm{D}$. It will be noted that the value of this angle computed by Einthoven's method differed from the estimate of the correct value by no more than 4 degrees for Positions $\mathrm{A}$ and $\mathrm{B}$ and by no more than 14 degrees for Position C. For Position D the difference was about 30 degrees. It was suspected that this large difference might be due to the gravitation of fluid into the more dorsal tissues, for it appeared when the plus electrode was thrust nearly three-fourths of the way through the chest.

The letter $(p)$ following the capital letter which designates the position of the input electrodes in Table I indicates that the leg electrode was just above the pubis; the letters $(s p)$, similarly used, indicate that, in addition, the two arm electrodes were on the anteromedial aspect of the shoulder joints. It will be noted that these changes in the positions of the electrodes had only trivial consequences. At the end of the experiment the distances from the three electrodes to the points where the brass rods penetrated the chest wall were measured. The distances of the right arm, left arm, and leg electrodes from the minus rod were $24,18.5$, and $37 \mathrm{~cm}$, respectively. The distances of the same electrodes from the plus rod were $20.5,16$, and $41.5 \mathrm{~cm}$., respectively. Since the electrodes were in the same position when Tracing 19 was taken as when Tracings 20 and 21 were made, these measurements throw no light upon the differences between Position $\mathrm{C}$ and Position $\mathrm{D}$ in respect to the magnitudes of the deflections in the limb leads.

Our chief purpose in making these observations was to ascertain whether Lead $V_{\mathrm{B}}$ would make it possible to measure the sagittal component of the electromotive force. Let us assume that the electrical field set up in the trunk by the voltage impressed on the input electrodes was equivalent to that produced by a centric doublet in a homogeneous spherical conductor. We may, then, consider the limb electrodes and the back electrodes the apices of a tetrahedron inscribed in a conducting sphere. If we regard this tetrahedron as equilateral, the distance between the plane defined by the limb leads and the doublet is equal to one-third of the radius of the hypothetical spherical surface. It may be shown 
WILSON ET AL.: SUBSTITUTION OF TETRAHEDRON FOR EINTHOVEN TRIANGLE 599

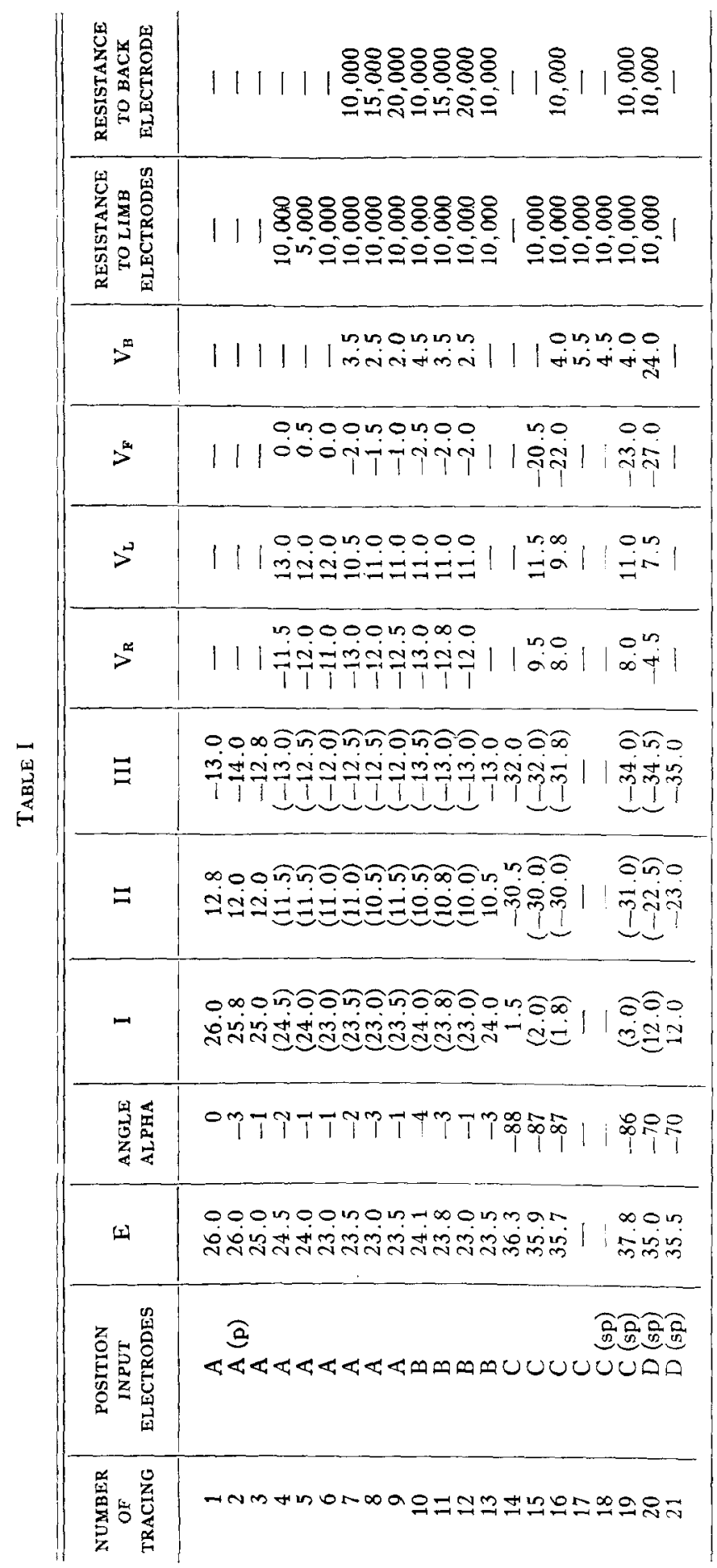


that if $E$ is the manifest magnitude of the component of the doublet that is parallel to the frontal plane, and if $\beta$ is the angle between this plane and the axis of the doublet, the deflection in Lead $V_{\mathrm{B}}$ should be $1 / 4 \sqrt{6} \mathrm{E}$ multiplied by tangent $\beta$. We are here supposing that the resistances connecting the central terminal to the four electrodes are equal.

On the other hand, we may suppose that the plane of the limb leads passes through the center of the spherical surface and, therefore, through the doublet. It may be shown that, in this case, the deflection in Lead $V_{B}$ should be $\frac{E}{\sqrt{3}}$

multiplied by tangent $\beta$, when the central terminal is connected to the limb electrodes only. Before we compare these theoretical values for the deflection in Lead $V_{B}$ with the values obtained experimentally, it is necessary to point out that the effect of removing the resistance between the terminal and the back electrode, or of making this resistance a multiple of the three equal resistances joining the terminal to the limb electrodes, may be easily computed. If we start with four equal resistances and disconnect the back electrode from the central terminal, without disturbing the other connections, the deflection in Lead $V_{B}$ should increase by one-third of its original value. If we double the resistance between the back electrode and the terminal instead of removing it, the deflection in Lead $V_{B}$ should increase one-seventh of its original value; if we increase this resistance by 50 per cent, the size of the deflection in Lead $V_{B}$ should increase one-eleventh of its original value. The experimental data are not in accord with these predictions, but they are too few and the changes in the size of the deflection concerned are too small to be regarded as decisive. The predictions involve simple algebraic calculations and Kirchkoff's second law, and can, therefore, hardly be at fault.

It will be seen at once that the deflections recorded in Lead $V_{B}$ are all much smaller than anticipated. Since the two hypotheses with regard to the position of the plane of the three leads with respect to the centric doublet lead to only minor differences in the theoretical size of the deflections in this lead, it will suffice if the values given in Table I, modified in each instance to the extent necessary to eliminate the effect of the resistance inserted between the central terminal and the back electrode, are compared with the value of $\frac{E}{\sqrt{3}}$ tangent $\beta$.

The latter is 8.3 for Position A, 13.9 for Position B, 21.1 for Position C, and 38 for Position D. Increasing the magnitude of the sagittal component by altering the relative depth of the two input electrodes always changed the size of the deflection in Lead $V_{B}$ in the proper direction, but the amount of the change did not bear a constant relation to that predicted. Position $B$ should have yielded a deflection 50 per cent larger than that given by Position $\mathrm{A}$. The actual increase in the size of the deflection was less than 30 per cent. Position D should have given a deflection about twice as large as Position $C$; it gave a deflection about six times as large.

We attribute the unexpectedly small values for the deflections in Lead $V_{B}$ obtained in this experiment to the gravitation of fluid into the more dorsal tissues. Whether this opinion is or is not well founded, only additional experiments of a 
similar kind can decide. It is not worth while at present to speculate regarding other possibilities. We may also call attention to another result that was unexpected. The rods used as input electrodes were parallel and always $5 \mathrm{~cm}$. apart, and they were always perpendicular to the anterior chest wall. The input voltage was not varied. It is surprising, therefore, that the value of $E$ should be $25 \mathrm{~mm}$. for Positions $\mathrm{A}$ and $\mathrm{B}$ and about $35 \mathrm{~mm}$. for Positions C and D. Unfor tunately, neither the resistance between the input electrodes nor the magnitude of the current flowing between them was measured. A lower resistance, and consequently a larger current, would be expected to increase the value of $E$, even though the voltage applied remained constant. In the absence of such measurements, we cannot explain satisfactorily why $E$ had one value when the frontal component of the input voltage was parallel to Lead I and another when it was roughly perpendicular to this lead.

\section{SUMMARY}

In an experiment on a cadaver, a potential difference was rhythmically impressed upon two small electrodes thrust into the heart or its immediate neighborhood.

The resulting differences in potential between a central terminal and four electrodes connected to it through equal resistances were recorded with the string galvanometer. The four electrodes were on the two arms, the left leg, and the left interscapular region.

By assuming that the electrical field generated in the trunk was equivalent to that of a centric doublet in a homogeneous spherical conductor and that the four electrodes were at the apices of a tetrahedron inscribed in this sphere, the experimental and the theoretical amplitudes of the deflections in the four leads could be compared. In general, it may be said that, with one exception, the deflections in the limb leads had the relative magnitudes expected. The deflections in the lead from the back were much smaller than anticipated. The last result is attributed to circumstances peculiar to the single experiment performed.

\section{APPENDIX}

Proposed Method of Finding the Spatial Electrical Axis.--The field of an clcctric dipole of moment $M$, located at the center, $O$, of a homogeneous conducting sphere of radius, $R$, is given by the equation

$$
V_{P}=M \cos \theta\left(1 / r^{2}+2 r / R^{3}\right)
$$

where $V_{\mathbf{P}}$ is the potential at the point $P, r$ is the length of the line $O P$, and $\theta$ is the angle made by this line with the axis of the dipole. When $P$ is on the surface of the sphere this equation reduces to

$$
V_{P}=A \cos \theta \quad \text { where } A=3 M / R^{2} .
$$

It may be pointed out that $\cos \theta$ of this equation is equal to the sum of the products formed by multiplying each of the direction cosines of the line $O P$ by the corresponding direction cosine of the axis of the dipole. 
Let $R, L, F$, and $B$ be the apiccs of an equilateral tetrahedron inscribed within the sphere. Take the center of the sphere, $O$, as origin, and take as $\mathrm{X}$ axis the line parallel to $R L$, as $\mathrm{Y}$ axis the line parallel to the perpendicular bisector of $R L$ which passes through $F$, and as $Z$ axis the line $O B$. The lines from $O$ to the apices of the tetrahedron will then have the direction cosines listed as follows:

$\begin{array}{cccc} & X \text { AXIS } & Y_{\text {AXIS }} & Z \text { AXIS } \\ \text { OR } & -\frac{1}{3} \sqrt{6} & -\frac{1}{3} \sqrt{2} & -\frac{1}{3} \\ \text { OL } & \frac{1}{3} \sqrt{6} & -\frac{1}{3} \sqrt{2} & -\frac{1}{3} \\ \text { OF } & 0 & \frac{2}{3} \sqrt{2} & -\frac{1}{3} \\ \text { OB } & 0 & 0 & 1\end{array}$

Let the axis of the dipole have the direction cosines $a, b$, and $c$. The potentials of the apices of the tetrahedron will then be given by the following equations:

$$
\begin{aligned}
& V_{\mathrm{R}}=\mathrm{A} \\
& \mathrm{V}_{\mathrm{L}}=\mathrm{A} \\
& \mathrm{V}_{\mathrm{F}}=\mathrm{A}
\end{aligned}
$$$$
\left\{\begin{array}{c}
-\frac{1}{3} \sqrt{6} a \\
\frac{1}{3} \sqrt{6} a \\
\end{array}\right.
$$$$
\begin{array}{rll}
-\frac{1}{3} & \sqrt{2} & b \\
\frac{1}{3} & \sqrt{2} & b \\
\frac{3}{3} & \frac{2}{2} & b
\end{array}
$$

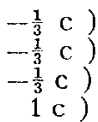

The dipole may be regarded as the vector sum of two components, one of which is parallel to the plane defined by the points $R, L$, and $F$, and the other coincident with the line $O B$. The first of these components will have no effect upon the potential at $B$, and the second will have identical effects upon the potentials of $R, L$, and $F$. We may split $A$ into two parts in the same way and define these two parts by the following equations:

$$
\begin{aligned}
& A_{f}=\sqrt{(A a)^{2}+(A b)^{2}} \\
& A_{s}=A C=V_{B}
\end{aligned}
$$

$A_{\mathrm{f}}$ bears the same relation to the first component of the dipole, and $A_{\mathrm{s}}$ the same relation to the second component, that $A$ bears to the dipole itself. $A_{\mathrm{f}}$ may be expressed in terms of Einthoven's $E$ derived from the potential differences between $R, L$, and $F$, regarded as the apices of the Einthoven triangle. We have

$$
\begin{aligned}
& \mathrm{E}^{2}=\left(\mathrm{V}_{\mathrm{L}}-\mathrm{V}_{\mathrm{R}}\right)^{2}+\frac{1}{3}\left[\left(\mathrm{~V}_{\mathrm{F}}-\mathrm{V}_{\mathrm{R}}\right)+\left(\mathrm{V}_{\mathrm{F}}-\mathrm{V}_{\mathrm{L}}\right)\right]^{2} \\
& \left(\mathrm{~V}_{\mathrm{L}}-\mathrm{V}_{\mathrm{R}}\right)=\frac{2}{3} \sqrt{6} \mathrm{Aa} \\
& \left(\mathrm{V}_{\mathrm{F}}-\mathrm{V}_{\mathrm{R}}\right)+\left(\mathrm{V}_{\mathrm{F}}-\mathrm{V}_{\mathrm{L}}\right)=2 \sqrt{2} \mathrm{Ab}
\end{aligned}
$$

Hence $\mathrm{E}^{2}={ }_{9}^{24}\left[(\mathrm{Aa})^{2}+(\mathrm{Ab})^{2}\right]=\frac{24}{9}\left(\mathrm{~A}_{\mathrm{f}}\right)^{2} \quad$ or $\quad \frac{1}{4} \sqrt{6} \mathrm{E}=\mathrm{A}_{\mathrm{f}}$

Since $A_{B}=V_{B}$, we have then

$$
A=\sqrt{A_{f}^{2}+A_{s}^{2}}=\sqrt{\frac{3}{8} E^{2}+V_{B}^{2}}
$$

The angle made by the axis of the dipole with the plane of $R L F$ may be found by the formula, $\cot \beta=\frac{1}{4} \sqrt{6} \mathrm{E} / \mathrm{V}_{\mathrm{B}}$.

It will be noted that $V_{R}+V_{L}+V_{F}+V_{B}=0$ and if electrodes at the points $R, L, F$, and $B$ are connected to a central terminal through equal resistances, this terminal will be at zero potential for all values of $a, b$, and $c$. The four leads 
from the central terminal to the four electrodes will, therefore, record the potentials $V_{\mathrm{R}}, V_{\mathrm{L}}, V_{\mathrm{F}}$, and $V_{\mathrm{B}}$.

Let us suppose that the points $R, L$, and $F$ are the apices of the triangle inscribed in the great circle represented by the intersection of the $X Y$ plane with the spherical surface, and that $B$ is the point where the positive half of the $Z$ axis penetrates this surface. The potentials of the four apices of the tetrahedron $R L F B$ will then be given by the equations:

$$
\begin{aligned}
& V_{\mathrm{R}}=\mathrm{A}(-1 / 2 \sqrt{3} \mathrm{a}-1 / 2 \mathrm{~b}) \\
& \mathrm{V}_{\mathrm{L}}=\mathrm{A}(1 / \sqrt{3} \mathrm{a}-1 / 2 \mathrm{~b}) \\
& \mathrm{V}_{\mathrm{F}}=\mathrm{Ab} \\
& \mathrm{V}_{\mathrm{B}}=\mathrm{Ac} \\
& \text { Here } V_{\mathrm{R}}+\mathrm{V}_{\mathrm{L}}+\mathrm{V}_{\mathrm{F}}=0, \mathrm{E} / \sqrt{3}=\mathrm{A}_{\mathrm{f}}, \text { and } \mathrm{V}_{\mathrm{B}}=\mathrm{A}_{\mathrm{s}}=\mathrm{Ac} \\
& \mathrm{A}^{2}=\mathrm{A}_{\mathrm{f}}{ }^{2}+\mathrm{A}_{\mathrm{s}}{ }^{2}=1 / 3 \mathrm{E}^{2}+\mathrm{V}_{\mathrm{B}}{ }^{2} \text { and } \cot \beta=(1 / \sqrt{3})\left(\mathrm{E} / \mathrm{V}_{\mathrm{B}}\right)
\end{aligned}
$$

The potential at the apices of the tetrahedron are recorded by leading to the electrodes at its apices from a central terminal connected through equal resistances to the electrodes at $R, L$, and $F$, but not to the electrode at $B$.

Method of Computing the Effect of Varying the Resistance Between the Central Terminal and the Electrode at B.-If the potentials of the four electrodes at $R, L, F$, and $B$ are $V_{\mathrm{R}}, V_{\mathrm{L}}, V_{\mathrm{F}}$, and $V_{\mathrm{B}}$, respectively, and the potential of the central terminal is $V$, we have for equal resistances, remembering that the sum of the currents meeting at a point is zero (Kirchkoff's second law).

$$
\begin{aligned}
& \left(V_{R}-V\right)+\left(V_{L}-V\right)+\left(V_{F}-V\right)+\left(V_{B}-V\right)=0 \\
& V=1 / 4\left(V_{R}+V_{L}+V_{F}+V_{B}\right) \\
& V_{B}-V=34 V_{B}-1 / 1\left(V_{R}+V_{L}+V_{F}\right)
\end{aligned}
$$

When the resistance between the central terminal and the electrode at $B$ is twice as large as the others we have

$$
\begin{aligned}
& \left(V_{R}-V\right)+\left(V_{L}-V\right)+\left(V_{F}-V\right)+1 / 2\left(V_{B}-V\right)=0 \\
& V_{R}+V_{L}+V_{F}+1 / 2 V_{B}=\frac{7}{2} V \\
& V_{B}-V=\frac{6}{7} V_{B}-\frac{2}{7}\left(V_{R}+V_{L}+V_{F}\right) \\
& 4(a)-\frac{7}{6}(b)=V_{B}-1 / 3\left(V_{R}+V_{L}+V_{F}\right) \\
& 3(b)=\frac{8}{7}(a)={ }_{7}^{6}\left[V_{B}-1 / 3\left(V_{R}+V_{L}+V_{F}\right)\right] \\
& \text { (b) }
\end{aligned}
$$

Note that $V_{B}-1 / 3\left(V_{R}+V_{L}+V_{F}\right)$ is the difference of potential between the central terminal and the electrode at $B$ when the connection between the terminal and this electrode is broken and the other connections are left undisturbed.

\section{REFERENCES}

1. Lewis, T., and Rothschild, M. A.: The Excitatory Process in the Dog's Heart. Part II. The Ventricles, Phil. 'Trans. Roy. Soc., Lond. sB 206:181, 1915.

2. Arrighi, Federico P.: El Eje Electrico del Corazon en el Espacio, Tesis del Doctorado en Medicina, Facultad de Ciencias Medicas, Universidad National de Buenos Aires. 1938. 